\title{
UNWINDING THE LONG ROAD THAT LEADS TO UNDERSTANDING AUTOIMMUNITY
}

\author{
Florencia RosetTI AND José C. CRISPÍN*
}

Department of Immunology and Rheumatology, Instituto Nacional de Ciencias Médicas y Nutrición Salvador Zubirán, Mexico City, Mexico

\begin{abstract}
Systemic autoimmune diseases are complex clinical conditions that arise in genetically predisposed individuals as a result of the interplay between their immune system and their environment. In this perspective, we briefly discuss our current understanding of the pathogenesis of autoimmunity and indicate four research avenues whose exploration will bring us closer to resolving fundamental questions that remain unanswered in this enigmatic field. (REV INVEST CLIN. 2021;73(5):297-301)
\end{abstract}

Key words: Autoimmunity. Autoimmune diseases. Immune system.

The clinical heterogeneity displayed by systemic lupus erythematosus (SLE) and other rheumatic autoimmune diseases has always baffled clinicians and taunted scientists. What is the basis of such heterogeneity and how does it impact our conceptual understanding of disease and consequently, our approach to clinical questions related to diagnosis and therapeutic decisions?

As other clinical entities, systemic autoimmune diseases were initially defined based on common phenotypes. However, unlike other diseases, their etiology could not come to rest on the discovery of an external pathogen or a faulty molecule. The complex pathophysiology of autoimmunity has eluded a unifying theory and what we have in our hands is a group of heterogeneous diseases with ill-defined causes and long lists of unanswered questions.
The development of autoimmunity is facilitated in certain individuals, in particular, in those with a family history of autoimmune disease. This heritability has been well documented and quantified ${ }^{1}$ and exhibits three important features: (a) all autoimmune diseases have a strong genetic component; (b) a relatively large number of risk alleles are shared between multiple autoimmune conditions; and (c) the products of most autoimmune-associated genes are part of immunological pathways, in particular, T cell signaling, tumor necrosis factor (TNF) signaling, or innate immunity ${ }^{2}$. These features, along with the fact that in the great majority of cases the onset of autoimmune diseases occurs in adulthood, suggest that these conditions are the result of pathological responses mounted by the immune system as a reaction to environmental stimuli. In other words, alleles that confer genetic predisposition do not cause autoimmune
*Corresponding author:

José C. Crispín

E-mail: carlos.crispina@incmnsz.mx
Received for publication: 04-06-2021

Approved for publication: 08-06-2021

DOI: $10.24875 / R I C .21000294$

0034-8376 / (c) 2021 Revista de Investigación Clínica. Published by Permanyer. This is an open access article under the CC BY-NC-ND license (http://creativecommons.org/licenses/by-nc-nd/4.0/). 
disease, but rather imprint the immune system of individuals who will eventually develop autoimmune pathology with a biased behavior that may become pathogenic under certain situations. Because the function of the adaptive immune system is based on its capacity to expand and relocate clones of cells that have differentiated in response to encounters with pathogens, microbiota, and other environmental elements, it is reasonable to assume that the presence of risk-conferring alleles alters this adaptation in a manner which eventually becomes pathogenic in patients with autoimmune disease. Therefore, even though a predisposing genetic architecture represents the foundation of autoimmune disease, it is the skewed responses accrued over the years what result in a pathogenic system that loses immune tolerance and acquires noxious inflammatory capacities. In this sense, autoimmune disease represents a pathological state reached by the immune system after repeated biased responses to common situations. Considering this conceptual landscape, we are faced with at least four endeavors that will improve our understanding of autoimmunity and allow us to delineate new clinical and therapeutic avenues (Figure 1 ).

\section{TO DEFINE THE CONTRIBUTIONS OF EACH PATHWAY TO DISEASE PHENOTYPE}

Risk alleles contribute to disease development or expression through the modulation of gene transcription or, less frequently, by altering the sequence of the encoded protein ${ }^{3}$. Associations between genetic variants and disease phenotypes have been mapped to common single nucleotide polymorphisms (SNPs) that may be part of the disease-associated variant or merely serve as markers of nearby variants. Individually, these genetic elements confer a small risk for the development of disease and in most cases; it has been difficult to identify the mechanisms through which they contribute to pathology. Several reasons may explain this, but probably the most important aspect is the fact that regulatory variants exert their effect on restricted cell lineages, under specific cellular contexts ${ }^{4}$. In other words, if the regulatory function of an SNP (or another genetic variant) is sought in the wrong cell type or tested using the erroneous stimulus, it will not become apparent.
Another important issue is that in many cases, the effects of genetic variants are tested in cells from patients. This approach might seem reasonable, but may introduce the additional confounding elements that a chronic inflammatory disease imposes on cells. The effects of genetic variants are more cleanly explored in cells from genotyped healthy individuals ${ }^{5}$. Finally, the most complex aspect is how several variants, in particular, those that encode for genes whose products act on the same pathway, affect the phenotype of a given cell. Although each variant is expected to affect cell behavior in a particular manner, it is the combined effect of a number of variants what probably promotes aberrant cellular responses and disease development and thus, it is the repertoire of risk alleles inherited by an individual what shapes the expression of his or her disease ${ }^{6}$.

\section{TO IDENTIFY RELEVANT ENVIRONMENTAL STIMULI AND UNDERSTAND HOW THEY INTERACT WITH RISK ALLELES}

A key aspect for understanding how autoimmune diseases develop and to design strategies to prevent them in predisposed individuals, is to comprehend how the environment interacts with the immune system and, in particular, how environmental stimuli may trigger abnormal responses in cells that are genetically susceptible to autoimmunity. Epidemiological studies have associated a relatively large number of environmental factors with autoimmune diseases, but in most cases, the associations are weak. An important limitation of most studies is that they seek associations in patients defined by their diagnosis, but disregarding their repertoire of genetic variants. In these cases, genetic heterogeneity weakens the associations, since patients are not equally susceptible to environmental stimuli. An example of this concept is the relationship between smoking and autoimmune diseases. The presence of the major histocompatibility complex (MHC) class II allele HLA-DRB1*15:01 greatly increases the risk of developing multiple sclerosis $(\mathrm{OR} \sim 3)^{7}$. However, when a person carrying that HLA allele smokes, the risk soars to an OR of $\sim 13^{8}$. A $\beta$ chain amino acid motif contained in a few HLADRB1 alleles - the shared epitope - is strongly associated with rheumatoid arthritis (OR 5-6 in individuals with shared epitope in both HLA-DR alleles). 
However, smoking increases the risk of rheumatoid arthritis to $\sim 19$ in females and to $\sim 60$ in males when both HLA-DRB1 alleles contain the shared epitope?. These data illustrate how an environmental factor may have a huge impact on disease development when certain genetic elements are present and how the environment may affect everyone in a different manner. Therefore, epidemiological research should consider genetic risk factors to produce data that allow us to understand how individual variation impacts susceptibility to common environmental elements.

\section{TO UNDERSTAND HOW THE EVOLUTION OF THE IMMUNE SYSTEM IN A PATIENT CONTRIBUTES TO DISEASE}

The immune system adapts in response to antigenic stimuli (i.e., infections, vaccines, and microbiota) and to non-antigenic signals that indicate the presence of pathogens, cellular stress, as well as hormonal and neural status. At the cellular level, this adaptation is achieved by radical changes in metabolism, gene expression, and cell survival. At the systemic level, it is met with conspicuous modifications of the abundance and location of specific lymphoid clones. Therefore, the immune system of an individual is in a constant evolution driven by a large number of environmental cues.

Following productive activation, lymphocytes differentiate into memory cells that have fewer stringent activation requirements and some relocate to nonlymphoid tissues ${ }^{10}$. To some extent, these changes may confer susceptibility to autoimmune disease, because differentiation of effector and memory cells with pro-inflammatory capacities, and their deployment to key tissues, can facilitate the initiation and perpetuation of local autoimmune responses ${ }^{11}$. In addition, the development of a chronic autoimmune response implies that self-reactive lymphoid clones become activated and, importantly, that they do so in the presence of inflammatory signals. This is relevant, because chronic activation of lymphocytes in the presence of TNF (and perhaps other pro-inflammatory cytokines) can affect their susceptibility to apoptosis and facilitate their effector capacities ${ }^{12}$. This illustrates a concept which is sometimes neglected, that due to the adaptive nature of the immune system, a chronic inflammatory disease may promote in immune cells a pathogenic behavior, where systemic inflammation induces pro-inflammatory functions that can intensify and perpetuate inflammation, generating a vicious cycle ${ }^{12}$. Thus, once disease is initiated and established, it modifies the phenotype and function of immune and non-immune cells. Although these changes are usually regarded as secondary, they may become relevant. The establishment of tissue resident pro-inflammatory memory cells in target organs may represent the key element that fuels chronicity and/or relapses in tissues afflicted by autoimmune disease.

\section{TO ANALYZE IMMUNE CELLS THAT INFILTRATE TARGET ORGANS AND UNDERSTAND THEIR RELATIONSHIP WITH LOCAL NON-IMMUNE CELLS}

Naïve lymphocytes seek antigens in secondary lymphoid organs and then migrate to tissues where they exert effector functions or establish local immune memory. In contrast to neutrophils, which spend most of their short lifetime in the blood patrolling for inflamed tissues, blood lymphocytes are travelers, a mixture of naïve and activated cells on route to lymphoid or non-lymphoid tissues and thus their experimental interrogation must be cautiously interpret$\mathrm{ed}^{13}$. As in other immune activation processes, the initiation of autoimmune diseases occurs in secondary lymphoid organs, and the noxious effects of the activated self-reactive cells are expressed in target organs. Therefore, it is extremely important to study the phenotype and behavior of immune cells in the tissues where the pathogenic cells are recruited and cause disease ${ }^{14}$. New technology able to investigate minute quantities of tissue at a single-cell level has allowed researchers to begin to understand the biology of pathogenic cells located in target organs ${ }^{15}$. In addition, we have begun to understand that local parenchymal cells play an active role during disease development by modulating and responding to the presence of infiltrating inflammatory cells. For example, synovial fibroblasts dramatically change their phenotype in response to cytokines and other products of inflammatory cells, becoming key pathogenic players in the synovial tissue of patients with rheumatoid arthritis $^{16}$. Therefore, the cellular and molecular environment of target organs can determine the susceptibility of a particular tissue to develop local chronic 
Figure 1. Fundamental questions that will allow to understand the biology and heterogeneity of human autoimmune disease.

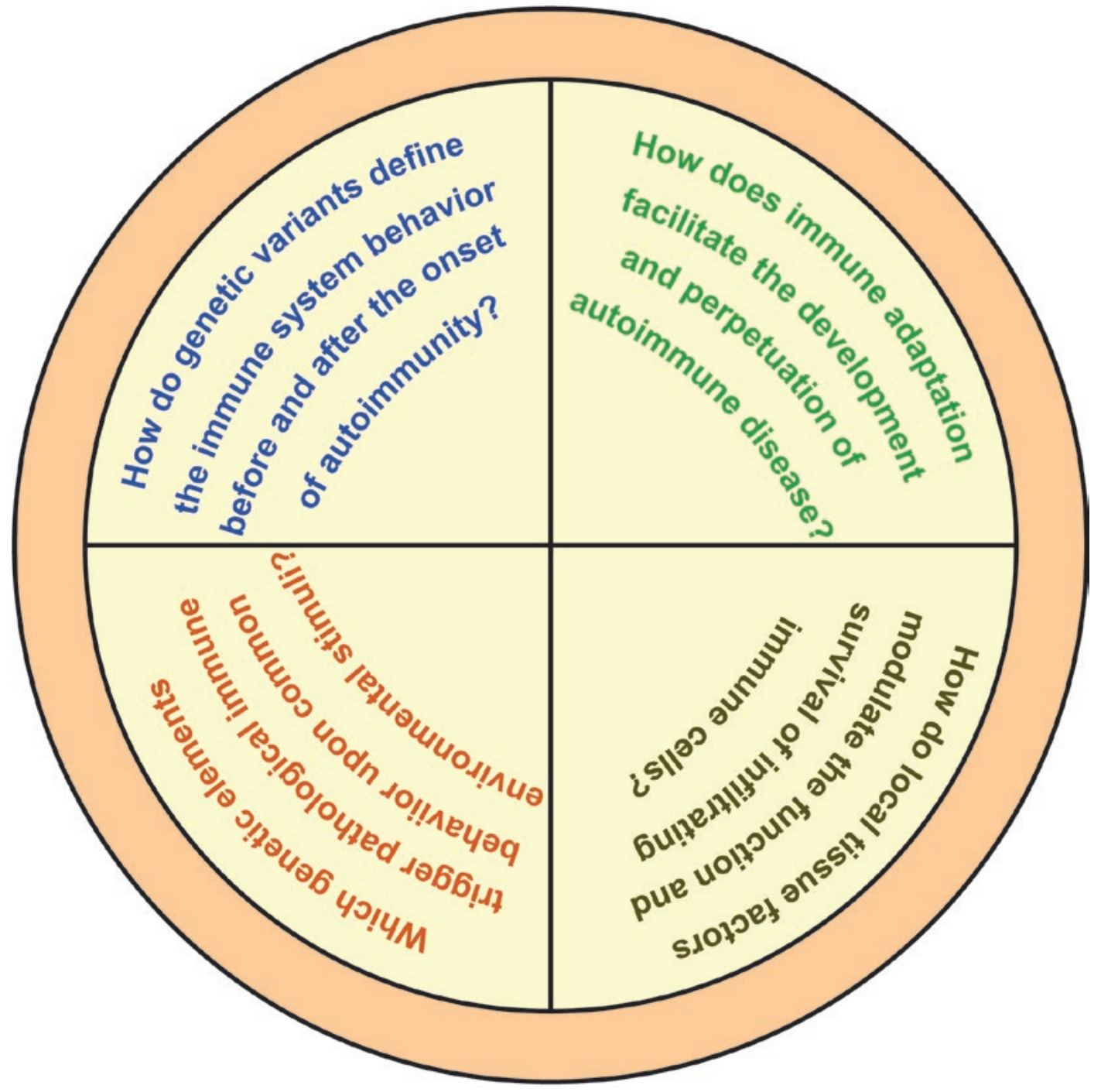

pathological inflammation, and this environment is shaped by immune cells (resident and infiltrating), by resident tissue cells, and importantly, by their interplay $^{17}$.

\section{CONCLUSIONS}

During the last decades, we have learned greatly about autoimmune diseases, in particular, about how patients can be grouped according to their phenotype. We have also understood that genetic factors and environmental triggers represent a mixture that allows the development of autoimmune pathology.
However, the concepts that have guided us to our current understanding cannot answer urgent questions that must be resolved to improve the way we conceptualize disease and, perhaps more importantly, the approach to design new therapies. Here, we have pointed at four avenues that may improve our understanding of these complex diseases.

\section{ACKNOWLEDGMENTS}

The work performed in the authors' laboratories was supported by the Consejo Nacional de Ciencia y Tecnología (CONACYT), Mexico City, Mexico, through 
grants FOSISS A3-S-39996 and Ciencia Básica A1S-34557 (FR), and FORDECYT 303046 (JCC).

\section{REFERENCES}

1. Li YR, Zhao SD, Li J, Bradfield JP, Mohebnasab M, Steel L, et al. Genetic sharing and heritability of paediatric age of onset autoimmune diseases. Nat Commun. 2015;6:1-10.

2. Zhernakova A, Van Diemen CC, Wijmenga C. Detecting shared pathogenesis from the shared genetics of immune-related diseases. Nat Rev Genet. 2009;10:43-55.

3. Maurano MT, Humbert R, Rynes E, Thurman RE, Haugen E, Wang $\mathrm{H}$, et al. Systematic localization of common disease-associated variation in regulatory DNA. Science. 2012;337:1190-5

4. Marson A, Housley WJ, Hafler DA. Genetic basis of autoimmunity. J Clin Invest. 2015;125:2234-41.

5. Gregersen PK, Klein G, Keogh M, Kern M, DeFranco M, Simpfendorfer KR, et al. The genotype and phenotype (GaP) registry: a living biobank for the analysis of quantitative traits. Immunol Res. 2015;63:107-12.

6. Barturen G, Beretta L, Cervera R, Van Vollenhoven R, AlarcónRiquelme ME. Moving towards a molecular taxonomy of autoimmune rheumatic diseases. Nat Rev Rheumatol. 2018:14:75-93.

7. Sawcer S, Hellenthal G, Pirinen M, Spencer CC, Patsopoulos NA, Moutsianas L, et al. Genetic risk and a primary role for cellmediated immune mechanisms in multiple sclerosis. Nature 2011:476:214-9.

8. Hedström AK, Sundqvist E, Bäärnhielm M, Nordin N, Hillert J, Kockum I, et al. Smoking and two human leukocyte antigen genes interact to increase the risk for multiple sclerosis. Brain. 2011;134:653-64

9. Klareskog L, Stolt P, Lundberg K, Källberg H, Bengtsson C Grunewald J, et al. A new model for an etiology of rheumatoid arthritis: smoking may trigger HLA-DR (shared epitope)-restricted immune reactions to autoantigens modified by citrullination. Arthritis Rheum. 2006:54:38-46.

10. Berard M, Tough DF. Qualitative differences between naïve and memory T cells. Immunology. 2002;106:127-38.

11. Krebs CF, Reimers D, Zhao Y, Paust HJ, Bartsch P, Nuñez S, et al. Pathogen-induced tissue-resident memory TH17 (TRM17) cells amplify autoimmune kidney disease. Sci Immunol. 2020;5:eaba4163.

12. Madera-Salcedo IK Sánchez-Hernández BE, Svyryd Y, EsquivelVelázquez M, Rodríguez-Rodríguez N, Trejo-Zambrano MI, et al. PPP2R2B hypermethylation causes acquired apoptosis deficiency in systemic autoimmune diseases. JCl Insight. 2019; 5:e126457.

13. Thome JJ, Yudanin N, Ohmura Y, Kubota M, Grinshpun B, Sathaliyawala $T$, et al. Spatial map of human $T$ cell compartmentalization and maintenance over decades of life. Cell. 2014; 159:814-28.

14. Farber DL. Tissues, not blood, are where immune cells function. Nature. 2021;593:506-9.

15. Arazi A, Rao DA, Berthier CC, Davidson A, Liu Y, Hoover PJ, et al. The immune cell landscape in kidneys of patients with lupus nephritis. Nat Immunol. 2019;20:902-14.

16. Huber LC, Distler O, Tarner I, Gay RE, Gay S, Pap T. Synovial fibroblasts: key players in rheumatoid arthritis. Rheumatology. 2006;45:669-75.

17. Liu K, Li QZ, Delgado-Vega AM, Abelson AK, Sánchez E, Kelly JA et al. Kallikrein genes are associated with lupus and glomerular basement membrane-specific antibody-induced nephritis in mice and humans. J Clin Invest. 2009;119:911-23. 